\title{
Surface Morphology associated with Phase Transformations in Indium-Rich Solid Solutions
}

\author{
By Yasumasa Koyama and Osamu Nittono
}

\begin{abstract}
The structural changes and surface morphology concerning phase transformations in indiumrich solid solutions, such as In-Tl, In-Cd, In-Pb, In-Sn and In-Hg, have been studied by means of crystallographic and metallographic methods. Four types of phase transformation are observed in indium-rich solid solutions as follows: (1) $f c c \rightleftarrows f c t(c / a>1),(2) f c c \rightleftarrows f c t(c / a<1),(3) f c t(c / a<1) \rightleftarrows$ $f c o \rightleftarrows f c t(c / a>1)$ and (4) $f c t(c / a<1) \rightleftarrows f c t(c / a>1)$. All the transformations, except the $f c o \rightleftarrows f c t(c / a>$ 1) one, are of martensitic type, and the alloy surface of low-temperature phase shows a surface relief associated with the phase transformation, which is ascribed to a banded structure due to $\{110\}$ transformation twinning. Under an assumption that these transformations take place in the form of a kind of the Bain lattice deformation, the lattice correspondence and the orientation relation between high- and low-temperature phases are well explained with respect to each phase transformation. The crystallographic orientation of the twin plane is explained on the basis of the Sapriel theory available for ferroelastic substances.
\end{abstract}

(Received July 30, 1982)

\section{Introduction}

Indium, which is a trivalent metal, has an $f c t$ structure with an axial ratio, $c / a$, of 1.076 . In indium-rich solid solutions with its neighbouring elements in the periodic table, such as $\mathrm{Cd}$ and $\mathrm{Hg}$ (divalent), $\mathrm{Tl}$ (trivalent), or $\mathrm{Sn}$ and $\mathrm{Pb}$ (tetravalent), there are characteristic phase changes depending upon the electron-atom ratio, $e / a^{(1)(2)}$. These phase changes in the indium-rich solid solutions have been studied by many workers. Tyzack and Raynor ${ }^{(3)}$ measured the axial ratio of the $f c t$ solid solutions by means of X-ray method, and showed that the axial ratio depends upon the electron-atom ratio and these phase changes are due to the electronic effect. Yonemitsu ${ }^{(4)}$ showed that the stability of the phases and the change in the axial ratio could be well explained on the basis of the nearly free electron model.

On the other hand, as the phase boundary between the neighboring phases is inclined against the temperature-axis in a phase diagram, the phase transformation is expected to occur when the temperature is changed. As for In-Tl alloys, Guttman ${ }^{(5)}$ showed that the alloys containing about $23 \mathrm{at} \% \mathrm{Tl}$ exhibit a phase transformation from the $f c c$ phase to the $f c t$ phase on cooling and the transformation is diffusionless, and that surfaces of the $f c t$ alloys show a banded structure due to $\{110\}$ transformation twinning ${ }^{(6)}$. It was also shown that the shape memory effect associated with the reverse transformation was observed ${ }^{(7)(8)}$. However, so far, the transformations except that in the In-Tl alloy have hardly been studied.

Recently, the present authors ${ }^{(9)-(17)}$ have investigated crystallographic and metallographic features of phase transformations in the indium-rich solid solutions, such as In-Tl, In- $\mathrm{Cd}, \mathrm{In}-\mathrm{Sn}, \mathrm{In}-\mathrm{Pb}$ and $\mathrm{In}-\mathrm{Hg}$, and already reported that these phase transformations are classified into four types as follows: (1) $f c c \rightleftarrows$ $f c t(c / a>1)$, (2) $f c c \rightleftarrows f c t(c / a<1)$, (3) $f c t(c / a<$ $1) \rightleftarrows f c o \rightleftarrows f c t(c / a>1)$ and (4) $f c t(c / a<1) \rightleftarrows f c t$ $(c / a>1)$. It was also shown that these alloys showed a shape memory effect associated with their reverse transformation, because all the phase transformations are thermoelastic martensitic. The purpose of the present paper is to report structural changes due to the phase transformations as well as the features of surface morphology in indium-rich solid solutions in a summarized form. The lattice correspondence between two phases which appear in each phase transformation is discussed in terms of the Bain-like lattice deformation. In addition, the surface morphology observed is discussed on the basis of the Sapriel theory ${ }^{(18)}$ which is applicable to ferroelastic materials ${ }^{(19)}$. 


\section{Experimental Procedure}

Indium-rich solid solutions were prepared from In, $\mathrm{Tl}, \mathrm{Cd}$ and $\mathrm{Pb}(99.99 \%$ purity), $\mathrm{Sn}$ $(99.999 \%)$ and $\mathrm{Hg}(99.9 \%)$. Predetermined weights of these metals were melted together in a vacuum $\left(10^{-3} \mathrm{~Pa}\right)$ in Pyrex crucibles. To ensure the uniformity of alloying composition, melting was repeated several times (usually 5 times). Resultant ingots, for which the losses in weight after melting were less than $0.1 \mathrm{wt} \%$, were homogenized for one week just below a melting point. Each of the resultant alloys was rolled into a sheet 0.2 to $0.5 \mathrm{~mm}$ thick at room temperature, and was subsequently held for a few hours just below the melting point. These sheets were used for the measurements of lattice parameters. The lattice parameters at
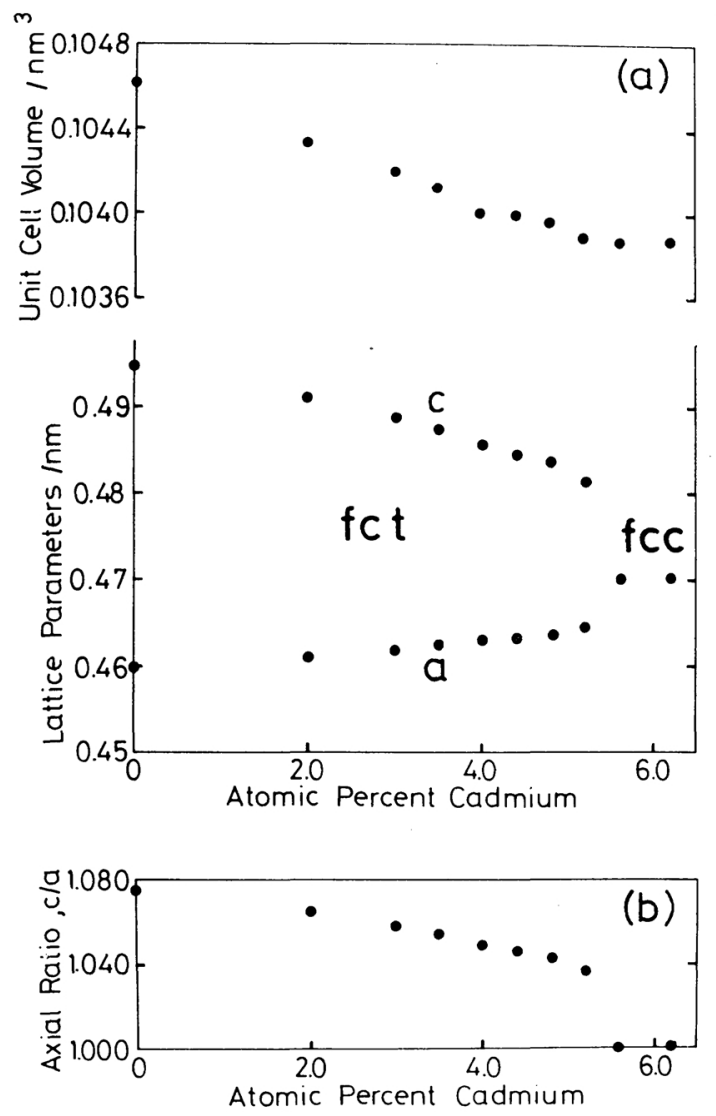

Fig. 1 (a) Lattice parameters and the unit cell volume and (b) the axial ratio $(c / a)$ as a function of at $\% \mathrm{Cd}$ at $298 \mathrm{~K}$, respectively. room temperature were measured by a conventional Debye-Scherrer technique. The measurement during phase transformation was undertaken by means of X-ray diffractometry with a heating stage, using $\mathrm{CuK} \alpha$ radiation. On the other hand, specimens used for observing a surface relief were prepared by a recrystallization method. The change in surface relief during phase transformation was observed by the aid of an optical and polarizing microscope with a heating stage. Structural changes due to phase transformation were also investigated by means of X-ray topography. Single crystals used for the topographic observations were prepared by a modified Bridgman method. Temperature fluctuations in the heating and cooling experiments were controlled within a temperature range of $\pm 1 \mathrm{~K}$. In order to observe a surface relief structure due to phase transformation, each alloy was chemically etched at a temperature in the high-temperature phase region by using the following solutions: (1) Solution of HF: $\mathrm{HNO}_{3}: \mathrm{CH}_{3} \mathrm{COOH}: \mathrm{H}_{2} \mathrm{O}=1: 2: 1: 12$ for In-Tl, In-Cd and In-Hg alloys, (2) solution of $\mathrm{H}_{2} \mathrm{O}_{2}: \mathrm{CH}_{3} \mathrm{COOH}: \mathrm{HCl}: \mathrm{H}_{2} \mathrm{O}=45: 50: 1: 4$ for $\mathrm{In}-\mathrm{Pb}$ alloys, and (3) solution of $\mathrm{HF}$ : $\mathrm{HNO}_{3}: \mathrm{HBr}=10: 10: 1$ for In-Sn alloys, where the composition ratio is given by the rate of volume.

\section{Experimental Results}

\section{The $f c c \rightleftarrows f c t(c / a>1)$ transformation $^{(9)-(11)}$ : In-TI, In-Cd and $\mathrm{In}-\mathrm{Hg}$ alloys}

In the In- $\mathrm{Tl}, \mathrm{In}-\mathrm{Cd}$ and $\mathrm{In}-\mathrm{Hg}$ alloys, the $f c t(c / a>1)$ phase changes into the $f c c$ one with increasing content of solute elements. Figure 1 shows the composition dependence of lattice parameters and of an axial ratio, $c / a$, in the In-Cd alloys. With increasing $\mathrm{Cd}$ content, the $a$-spacing increases and the $c$-spacing decreases, so that the axial ratio decreases toward unity and finally the $f c c$ phase appears at about $5.5 \mathrm{at} \% \mathrm{Cd}$. Characteristic feature of the phase changes observed in the In-Cd alloys was the same as that in the In- $\mathrm{Tl}$ and $\mathrm{In}-\mathrm{Hg}$ alloys. Structural change occurring during the $f c c \rightleftarrows f c t$ phase transformation was also examined by 
means of optical microscopy and X-ray topography. One example of a banded surface structure observed in all the $f c t$ alloys is shown in Photo. 1. An analysis of its Laue photographs showed that the banded structure is ascribed to $\{110\}$ transformation twinning. In addition, in-situ observation showed that the alloys undergo a martensitic phase transformation from $f c c$ to $f c t$ by the migration of a $f c c / f c t$ single interface on cooling. The twin boundary can be easily moved by applying a small stress. This indicates that these fct alloys are ferroelastic materials, according to Aizu's definition $^{(19)}$. Photograph 2 shows a series of X-ray topographs taken at various temperatures during the $f c t \rightarrow f c c$ reverse transformation of an In-21 at $\% \mathrm{Tl}$ alloy single crystal on heating. A topographic image of the $f c t$ alloy is given as black-white bands, because one variant of twin (I) is in the Bragg condition and the other (II) is out of condition. This shows that the inclination of the lattice planes of each variant is alternately the same. When the temperature is raised, a portion of the crystal exhibits a phase transformation from the $f c t$ to the $f c c$ phase at $329 \mathrm{~K}$, as shown in Photos. 2(b) and 2(c). The positional correspondence between these two topographs shows that the $f c c$ phase begins to nucleate along the twin boundaries and grows with increasing temperature. On further heating, the alloy shows a temperature range over which two phases coexist (see Photo. 2(d)) and finally becomes the $f c c$ single phase at $383 \mathrm{~K}$, as shown in Photo. 2(e). The observation of the $f c t \rightarrow f c c$ reverse transformation in the $\mathrm{In}-\mathrm{Cd}$ and $\mathrm{In}-\mathrm{Hg}$ alloys by means of optical microscopy also showed that a temperature range of the two-phase field was much narrower than that of the In-Tl alloy.

\section{The $f c c \rightleftarrows f c t(c / a<1)$ transformation: In-(28-36 at $\%) \mathbf{P b}$ alloys ${ }^{(13)}$}

The indium-lead alloys containing $28-36$ at $\%$ $\mathrm{Pb}$ exhibit a phase transformation from the $f c c$ phase to the $f c t(c / a<1)$ one on cooling. Figure 2 shows the lattice parameters and the axial ratio at various temperatures during the $f c c \rightleftarrows$ fct $(c / a<1)$ phase transformation of an In$32 \mathrm{at} \% \mathrm{~Pb}$ alloy. The $f c c$ phase becomes the fct one at $360 \mathrm{~K}$ on cooling, and the axial ratio

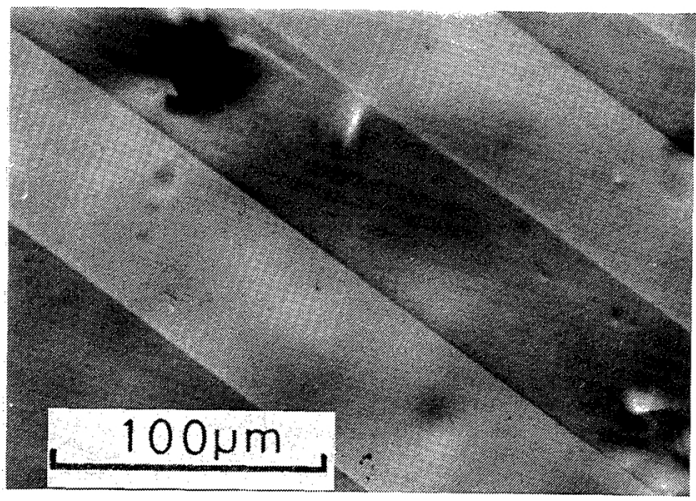

Photo. 1 Optical micrograph showing a banded surface relief structure due to $\{110\}$ transformation twinning of an In-5.2 at \% Cd alloy.

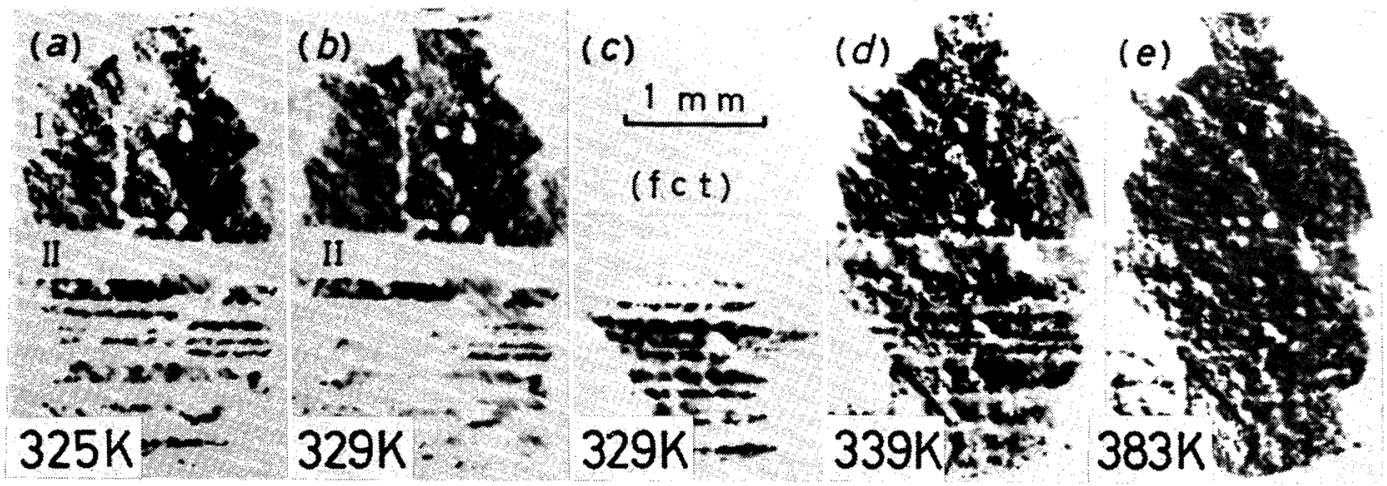

Photo. 2 X-ray topographs showing the structural changes on heating of an In- 21 at $\% \mathrm{Tl}$ single crystal.

Topographic images are somewhat spotty because of surface roughness caused by chemical etching at room temperature. 


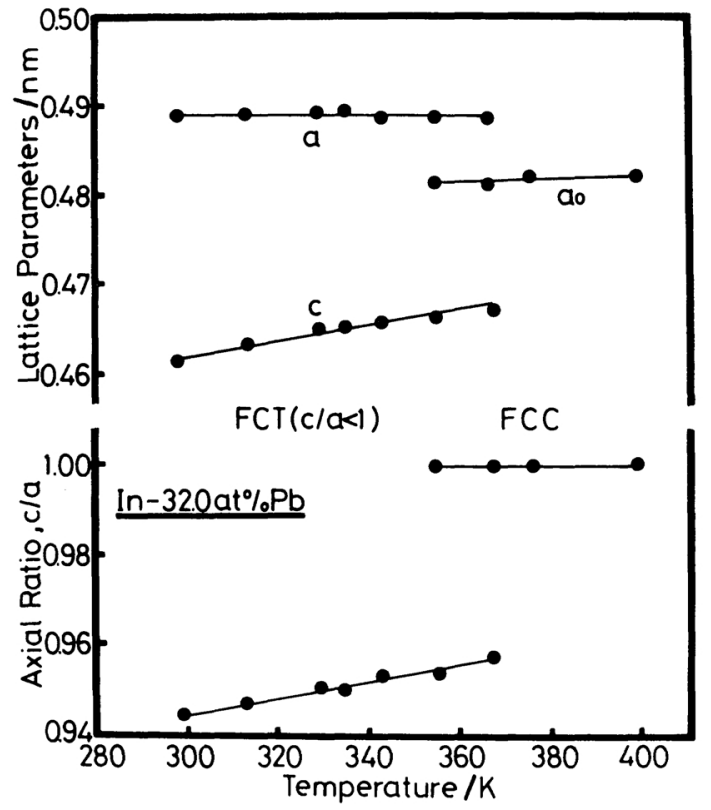

Fig. 2 Temperature dependence of lattice parameters and the axial ratio of an $\mathrm{In}-32.0 \mathrm{at} \% \mathrm{~Pb}$ alloy.

is 0.96 at the transformation point and decreases with falling temperature. The change in volume and the temperature hysteresis due to the phase transformation are estimated to be $0.3 \%$ and $15 \mathrm{~K}$, respectively. Phase transforma- tion behavior was also examined by means of optical microscopy. The change in surface relief structure in an In-32.0 at $\% \mathrm{~Pb}$ alloy, which was heated to a high-temperature phase region and subsequently cooled, is shown in Photo. 3(a). Crystal surfaces of the $f c t$ alloys reveal a banded surface structure. An analysis of the Laue photographs showed that the surface relief is also ascribed to $\{110\}$ transformation twinning. When the temperature is raised, each band disappears suddenly in a given grain at about $330 \mathrm{~K}$ (see Photo. 3(b)), and finally a smooth surface field due to the $f c c$ phase extends all over the grain (see Photo. 3(c)). In the $f c c \rightarrow f c t$ transformation on cooling, a band appears abruptly in a certain grain, and new bands parallel and adjacent to the existing bands are produced (Photo. 3(d)), and then the bands extend in the whole grain (Photo. 3(e)). These observations indicate that the transformation is a thermoelastic martensitic one. Another change in surface relief observed in the reverse transformation of an In- $34 \mathrm{at} \% \mathrm{~Pb}$ alloy is reproduced in Photo. 4. In this case, near the transformation temperature the boundary between two adjacent bands becomes less distinct on an optical photograph, and then the surface relief due to the band-shaped martensites dis-



Photo. 3 A series of optical micrographs showing a change in surface structure of an In$32 \mathrm{at} \% \mathrm{~Pb}$ alloy on heating and subsequent cooling. (a) $308 \mathrm{~K}$, (b) $330 \mathrm{~K}$, (c) $347 \mathrm{~K}$, (d) $313 \mathrm{~K}$, (e) $309 \mathrm{~K}$. 


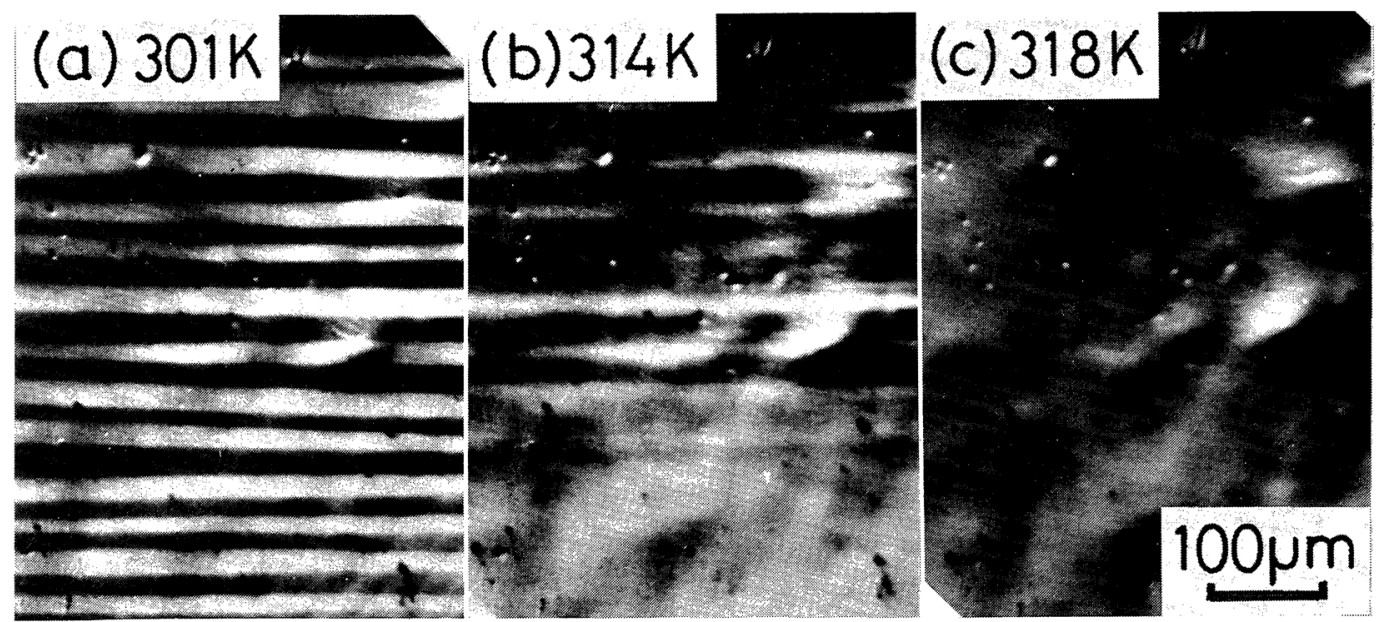

Photo. 4 Optical micrographs showing a change in surface structure of an In- 34 at $\% \mathrm{~Pb}$ alloy on heating.

appears (see Photo. 4(c)). This behavior is quite similar to that in the In-Tl alloy ${ }^{(5)}$.

\section{The $f c t(c / a<1) \rightleftarrows f c o \rightleftarrows f c t(c / a>1)$ transformation: In-(12-16 at $\%) P b$ alloys $^{(13)}$}

According to the phase diagram reported so far, an $f c t(c / a<1) \rightleftarrows f c t(c / a>1)$ phase transformation is expected to occur in In-(12-16 at \%) $\mathrm{Pb}$ alloys. Figure 3 represents a series of $\mathrm{X}$-ray 200 reflection profiles measured at various temperatures through the $f c t(c / a<1) \rightleftarrows f c t(c / a>$ 1) transformation in an $\mathrm{In}-12.5 \mathrm{at} \% \mathrm{~Pb}$ alloy. The profile measured at $293 \mathrm{~K}$ consists of two peaks, i.e. 200 and 002 peaks corresponding to

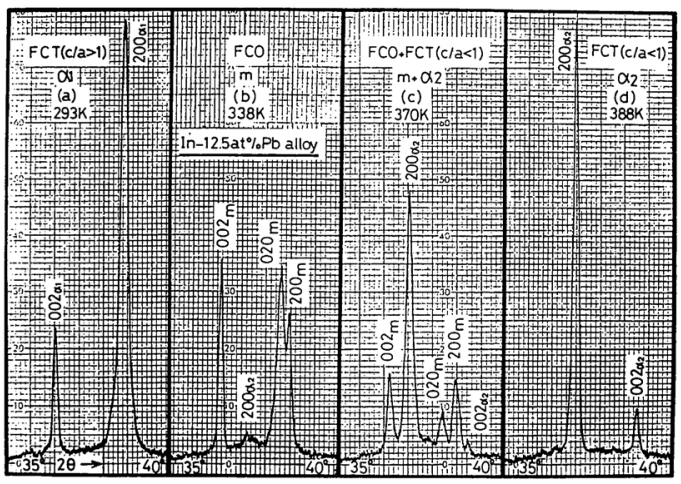

Fig. 3 A series of 220 X-ray reflection profiles from an In-12.5 at $\% \mathrm{~Pb}$ alloy on heating. The suffix $\mathrm{m}$ denotes a metastable intermediate phase. the $f c t(c / a>1)$ phase. On heating, the 200 peak splits into two peaks corresponding to 200 and 020 reflections, and the 002 peak shifts slightly. In addition, a small peak due to the $f c t(c / a<1)$ phase (a high-temperature phase) begins to appear, so that four peaks are observed on the reflection profiles (see Fig. 3(b)). The main three peaks are ascribed to the existence of a face centred orthorhombic lattice ( $f c o$ ) during the transformation. This was confirmed by an analysis of $111,220,311$ and 222 reflection lines observed in this experiment, although it is not reproduced here. With increasing temperature, the $f c o$ phase coexists with the $f c t(c / a<1)$ one in a narrow temperature range (Fig. 3(c)), and then the alloy becomes the $f c t(c / a<1)$ single phase, and finally only the $f c t(c / a<1)$ peaks are observed on the profile, as shown in Fig. 3(c). Figure 4 shows the temperature dependence of the lattice constants of an In$12.5 \mathrm{at} \% \mathrm{~Pb}$ alloy. The alloy undergoes the $f c t$ $(c / a<1) \rightleftarrows f c o$ phase transformation at $370 \mathrm{~K}$. The change in volume due to the phase transformation is $0.3 \%$ and the hysteresis of the transformation temperature is $15-20 \mathrm{~K}$, indicating that the phase transformation is of the first order. On further cooling the $a$ and $b$ spacings of the fco phase approach each other and become equal, so that the $f c o$ alloy is transformed into the $f c t(c / a>1)$ one. The change in volume due to the $f c o \rightarrow f c t(c / a>1)$ transforma- 


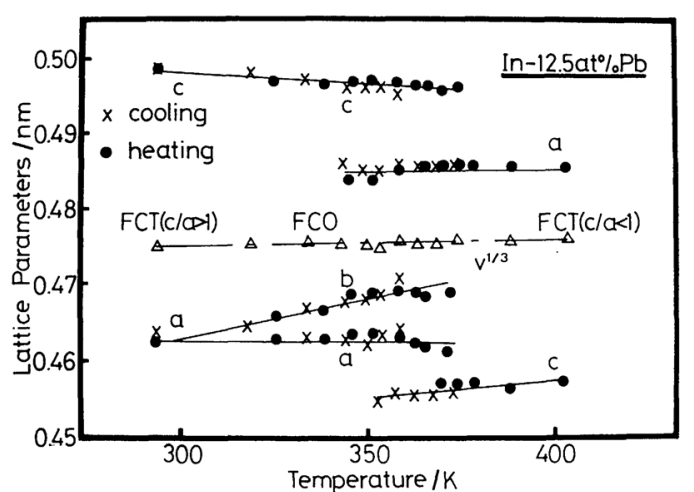

Fig. 4 Temperature dependence of lattice parameters of an In-12.5 at $\% \mathrm{~Pb}$ alloy.

tion could not be detected in this experiment. From these results, it is concluded that the $f c t(c / a<1) \rightleftarrows f c t(c / a>1)$ transformation should be rather expressed by the $f c t(c / a<1) \rightleftarrows f c o \rightleftarrows f c t$ $(c / a>1)$ transformation. The surface structure associated with the $f c t(c / a<1) \rightleftarrows f c o$ transformation was observed in an In-12.8 at $\% \mathrm{~Pb}$ alloy, as shown in Photo. 5, and the fine parallel bands observed in the fco phase were verified by the X-ray diffraction method to be $\{110\}$ twins. The change in surface relief was reversible on both cooling and heating processes. This shows that the $f c t(c / a<1) \rightleftarrows f c o$ transformation is thermoelastic martensitic. On the other hand, no appreciable change in surface relief is observed in the $f c o \rightleftarrows f c t(c / a>1)$ transformation.

\section{The $f c t(c / a<1) \rightleftarrows f c t(c / a>1)$ transformation: In-(13-15 at $\%)$ Sn alloys $^{(12)(15)}$}

The alloys containing $13-15 \mathrm{at} \% \mathrm{Sn}$ are expected to undergo an $f c t(c / a<1) \rightleftarrows f c t(c / a>1)$ phase transformation. Photographs 6(a) and 6(b) are optical photographs showing surface structures of In-13 at $\%$ Sn alloys. One (a) was obtained from the alloy cooled slowly to room temperature, and the other (b) from that quenched to the temperature of liquid nitrogen. Both the alloys were etched so as to have flat and smooth surfaces at a temperature in the high-temperature phase region. The former ex-

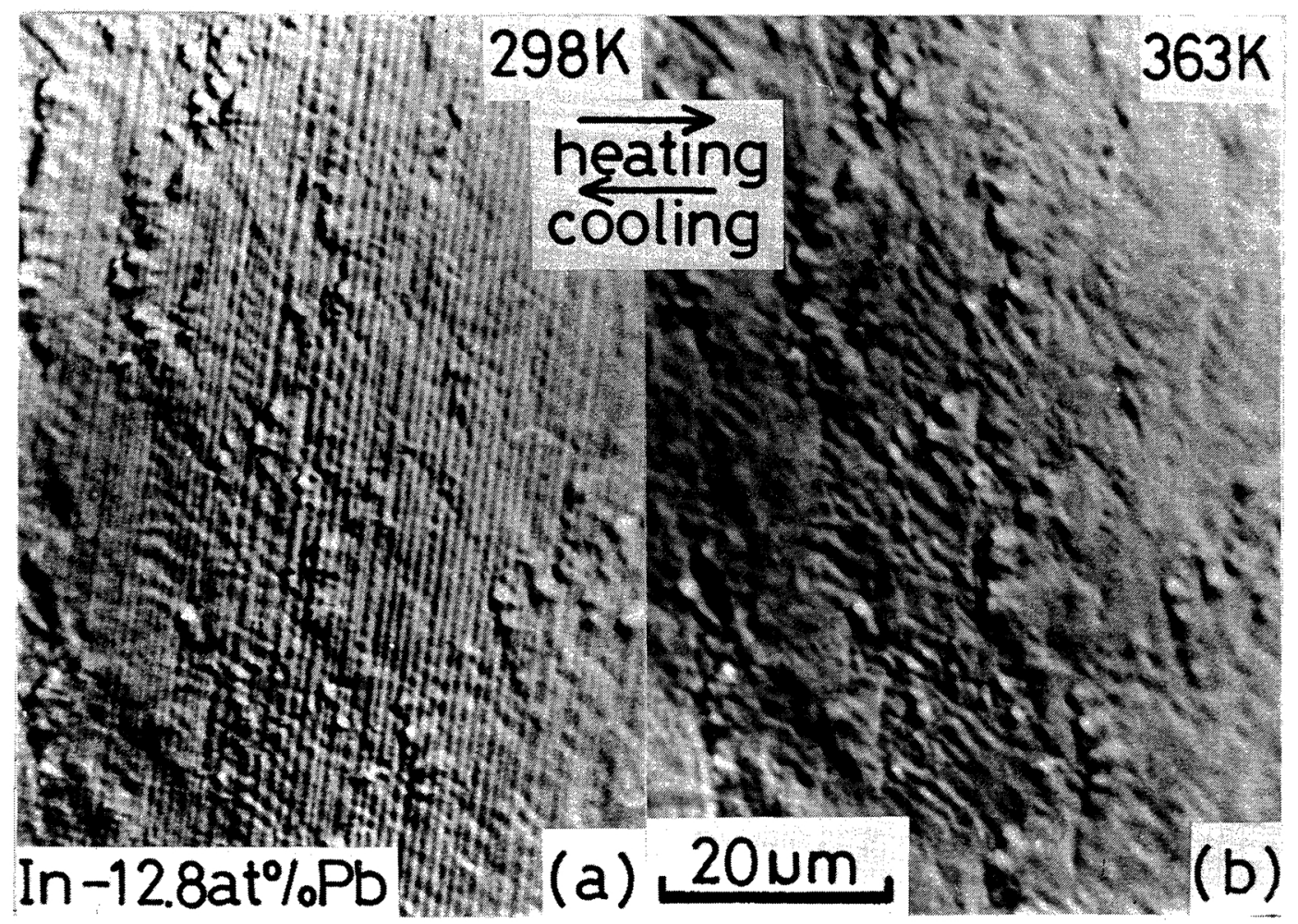

Photo. 5 Optical micrographs showing surface structures associated with the $f c t(c / a<1) \rightleftarrows f c o$ phase transformation. 

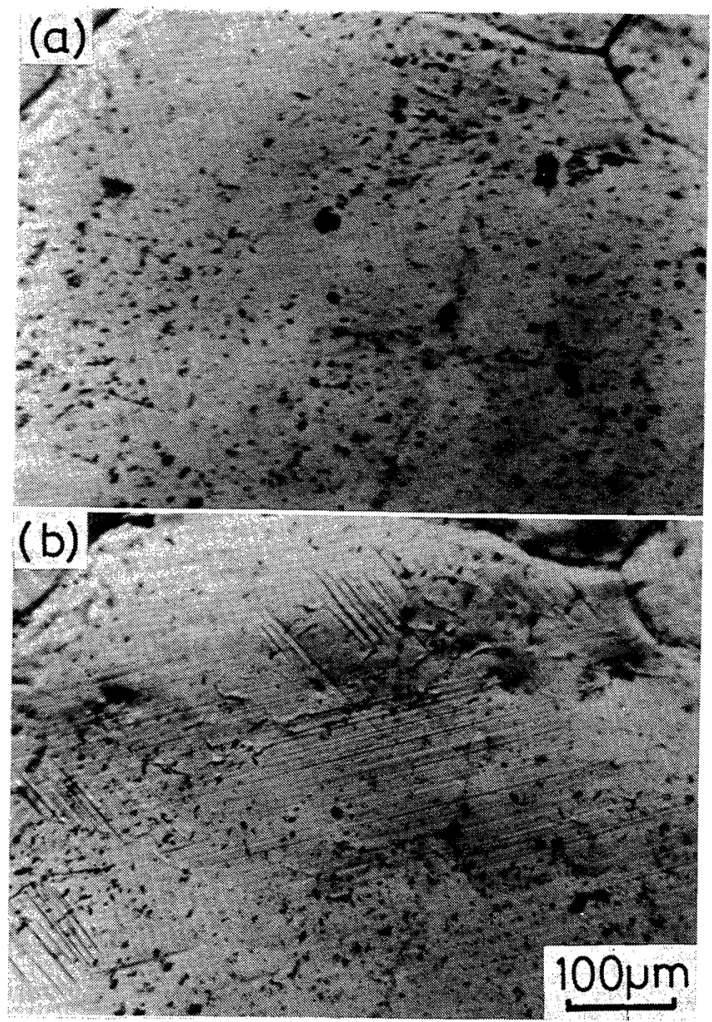

Photo. 6 Optical micrographs showing surface structures of an In-13 at \% Sn alloy. (a) Slowly cooled to room temperature in a furnace, (b) quenched into liquid nitrogen.

hibits a flat surface, being similar to that of the $f c t(c / a<1)$ phase, but the latter shows a banded relief structure. Further, the surface structures were examined by means of X-ray diffractometry. X-ray 220 profiles of an In-13 at $\% \mathrm{Sn}$ alloy are shown in Fig. 5. The quenched alloy shows only the profiles due to the $f c t(c / a>1)$ phase (Fig. 5(a)). On the other hand, the slowly cooled alloy retains an $f c t(c / a<1)$ phase, which is the high-temperature phase, and after having been kept at room temperature for 40 days, it shows the coexistence of both $f c t(c / a<1)$ and $f c t(c / a>1)$ phases (Fig. 5(b)). The result indicates that the In-Sn alloys undergo the $f c t$ $(c / a<1) \rightleftarrows f c t(c / a>1)$ martensitic transformation only when quenched into liquid nitrogen. The temperature dependence of both the lattice parameters and the axial ratio for an In-13 at \% Sn alloy on heating is shown in Fig. 6. The alloy exhibits a transformation from $f c t(c / a>1)$ to

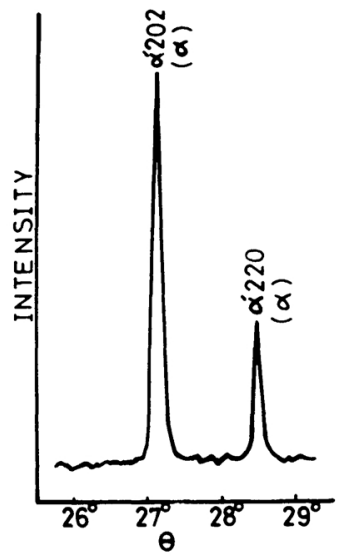

(a)

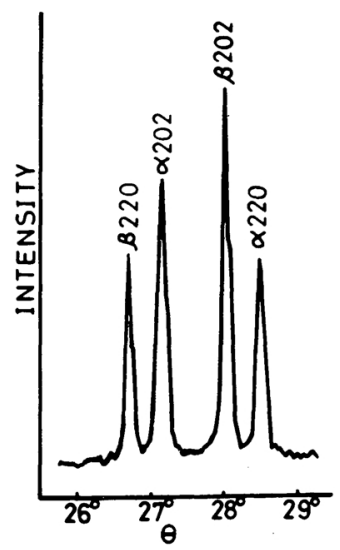

(b)
Fig. 5 X-ray reflection profiles from an In13.0 at $\% \mathrm{Sn}$ alloy. (a) Quenched from $403 \mathrm{~K}$ into liquid nitrogen, (b) kept at room temperature for 40 days.

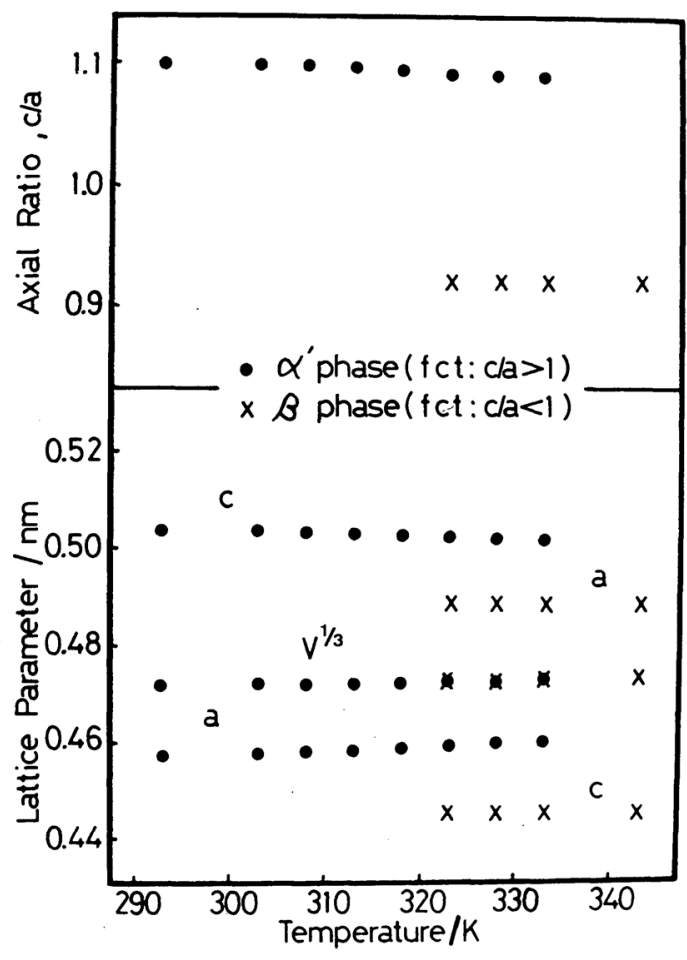

Fig. 6 Temperature dependence of lattice parameters, the axial ratio and the cube root of the unit cell volume of an In-13 at $\% \mathrm{Sn}$ alloy.

$f c t(c / a<1)$ at about $350 \mathrm{~K}$. The $a$ and $c$ parameters of both the fct phases hardly change with temperature, and thus the axial ratio is kept nearly constant. The jump of the axial ratio, 
$(1-c / a)$, and the volume change at the transformation temperature are 0.2 and $0.7 \%$, respectively.

Photographs 7(a) and 7(b) are two optical micrographs of an In-13.4 at $\%$ Sn alloy, which were taken from a quenched $f c t(c / a>1)$ alloy $\left(\alpha^{\prime}\right.$ phase) and from a reverted $f c t(c / a<1)$ one ( $\beta$ phase), respectively. The $M_{s}$ and $A_{s}$ points are respectively 273 and $318 \mathrm{~K}$. Therefore, for both alloys optical observations were made at $298 \mathrm{~K}$ within a short time. The quenched alloy shows a banded surface relief due to $\{110\}$ transformation twinning (T1), which is similar to that in the other indium-rich solid solutions, as observed in Photo. 7(a). In this case, when the alloy was heated above the $A_{s}$ point, the old bands were still found faintly and new bands (T2) suddenly appeared across the existing old ones (Photo. 7(b)). Figure 7 represents a stereographic projection obtained by an analysis of Laue photographs taken from the reverted $f c t(c / a<1)$ alloy. From this analysis, it is
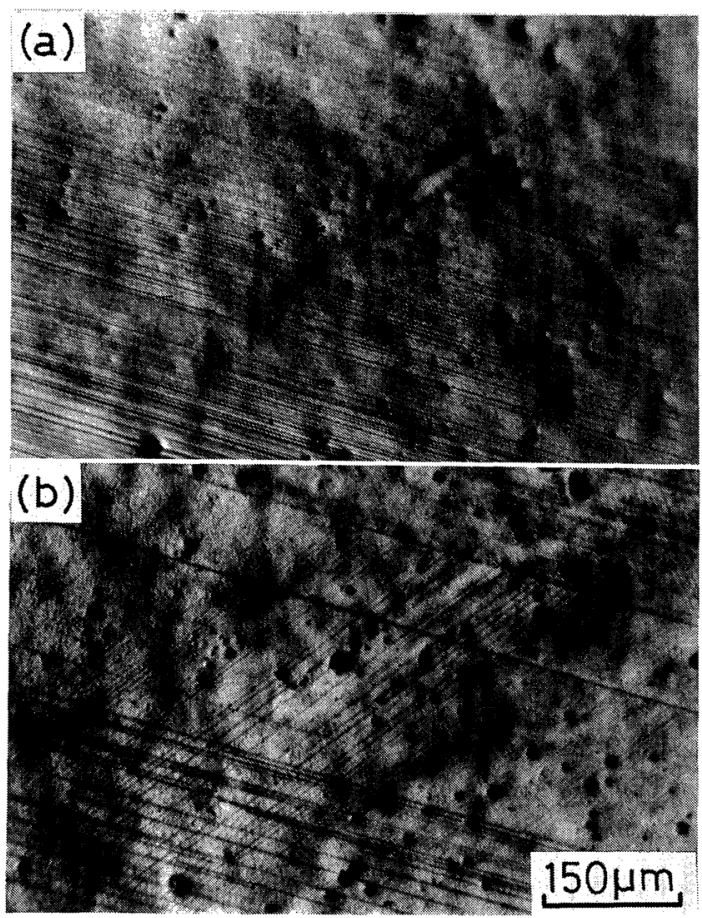

Photo. 7 Optical micrographs showing a banded surface relief of an In-13.4 at $\% \mathrm{Sn}$ alloy. (a) Quenched crystal ( $\alpha^{\prime}$ phase), (b) after the $\alpha^{\prime} \rightarrow \beta$ reverse transformation. concluded that the reverted $\beta$ phase crystal shows only the $f c t(c / a<1)$ phase and consists of two variants of this phase, which are expressed by open and closed marks in the stereographic projection, and that these variants have a twin relation with each other in reference to (110) planes.

\section{Discussion}

\section{Lattice correspondence of the phase transformations}

It has been shown that there are four kinds of phase transformation in the indium-rich solid solutions as follows;

(a) $f c c \rightleftarrows f c t(c / a>1):$ In- $(18-30$ at $\%) \mathrm{Tl}, \quad$ In$(4.0-5.5 \mathrm{at} \%) \mathrm{Cd}$ and $\mathrm{In}-$ $(6.8-7.0 \mathrm{at} \%) \mathrm{Hg}$ alloys,

(b) $f c c \rightleftarrows f c t(c / a<1):$ In- $(28-36$ at $\%) \mathrm{Pb}$ alloys,

(c) $\quad f c t(c / a<1) \rightleftarrows f c o \rightleftarrows f c t(c / a>1)$ : In-(12$16 \mathrm{at} \%) \mathrm{Pb}$ alloys,

(d) $f c t(c / a<1) \rightleftarrows f c t(c / a>1):$ In- $(13-15$ at $\%)$ Sn alloys.

These phase transformations, except the $f c o \rightleftarrows$ $f c t(c / a>1)$ one in the case (c), are martensitic in nature.

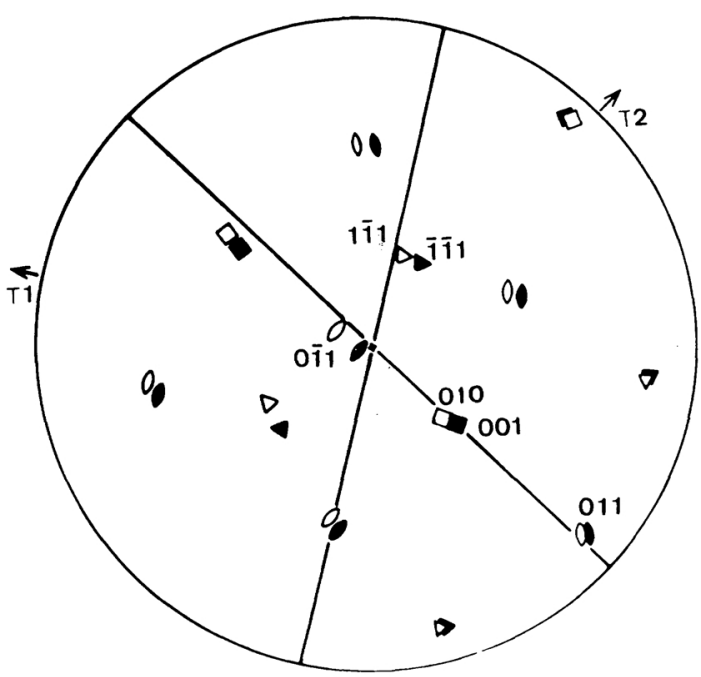

Fig. 7 Stereographic projection of the $\beta$ phase crystal $(c / a=0.92)$ which was retransformed from the $\alpha^{\prime}$ phase. The arrows $T_{1}$ and $T_{2}$ denote the directions of the traces of the $\{110\}$ twins shown in Photo. 7. 
We consider the lattice correspondence between two adjacent phases which appear in each transformation. According to the same treatment as that mentioned in the previous papers $^{(10)(16)}$, when three axial lengths along the $a, b$ and $c$ directions of the $f c c$ lattice are discribed as $(a, b, c)=(1,1,1)$, the axial lengths of the $f c t(c / a>1)$ one, for example, can be represented as $(1-\varepsilon, 1-\varepsilon, 1+2 \varepsilon)=(a, a, c)$, where $\varepsilon$ is a spontaneous strain concerning the phase transformation and $\varepsilon^{2}$ is assumed to be smaller than unity and negligible. This shows that the $f c c \rightleftarrows f c t(c / a>1)$ lattice transformation appears to correspond to a change in axial length given by $(1,1,1) \rightarrow(1-\varepsilon, 1-\varepsilon, 1+2 \varepsilon)$. In other words, this suggests that the lattice transformation associated with the phase transformation takes place in the form of the Bain lattice distortion in the sence that the $a$ and $b$ spacings contract by an amount of $\varepsilon$ and the $c$ spacing expands by $2 \varepsilon$ as a result of phase transformation. Therefore, in what follows, each phase transformation is discussed on the basis of the Bain lattice deformation.

In view of the axial correspondence in the $f c c \rightleftarrows f c t(c / a>1)$ lattice transformation, each axis of the $f c c$ lattice can become the $c$-axis of the $f c t$ one after phase transformation. Thus the orientation relation between two phases is $\langle 001\rangle_{f c c} / /[001]_{f c t}$. Accordingly, the $f c t$ martensite can have three variants, as shown in Fig. 8. However, after the reverse transformation the $f c c$ phase can have only a single variant

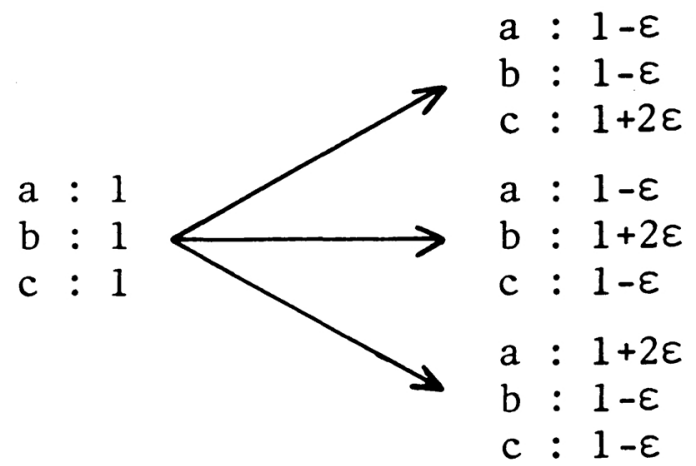

fcc $\quad \vec{\leftarrow} \quad \operatorname{fct}(\mathrm{c} / \mathrm{a}>1)$

Fig. 8 Lattice correspondence in the $f c c \rightleftarrows f c t(c / a>1)$ transformation. regardless of each variant of the fct lattice. This indicates that the crystallographic reversibility during the phase transformation is preserved and the shape memory effect is revealed. Next, we treat the $f c c \rightleftarrows f c t(c / a<1)$ lattice transformation, where the $f c t(c / a<1)$ lattice is given as $(1+\varepsilon, 1+\varepsilon, 1-2 \varepsilon)$ and thus the axial ratio is $c / a=(1-2 \varepsilon) /(1+\varepsilon)=1-3 \varepsilon<1$. In this case, as a result of lattice transformation, the $a$ and $b$ spacings of the $f c c$ lattice expand by $\varepsilon$, while the $c$ spacing contracts by $2 \varepsilon$, and thus we have a relation of $\langle 001\rangle_{f c c} / /[001]_{f c t}$. As in the analogous $f c c \rightleftarrows f c t(c / a>1)$ lattice transformation, there are three variants which are possible to exist in the fct lattice, as shown in Fig. 9. The three $f c t$ variants can be converted into a single variant of the $f c c$ lattice after the reverse transformation, and thus the shape memory effect is also expected. In the case (c), where the $f c t$ $(c / a<1)$ lattice is first deformed into an $f c o$ one and is further deformed into the $f c t(c / a>1)$ lattice with falling temperature, a change in axial length during the $f c t(c / a<1) \rightleftarrows f c o$ lattice transformation is given by a relation $(a, b, c)=$ $(a, a, c)=(1+\varepsilon, \quad 1+\varepsilon, \quad 1-2 \varepsilon) \rightarrow(1+2 \varepsilon, \quad 1-\delta$, $1-2 \varepsilon+\delta)=(c, b, a)$. In other words, the changes in axial length along the $a, b$ and $c$ directions of the $f c t$ lattice are given by $+\varepsilon$, $-\varepsilon-\delta$ and $+\delta$, respectively, where $\delta$ is a little smaller than $\varepsilon$. In an In- $12.8 \mathrm{at} \% \mathrm{~Pb}$ alloy shown in Fig. 4, $\varepsilon$ and $\delta$ were 0.019 and 0.015 , respectively. The orientation relation between the $f c o$ and $f c t$ lattices is $[001]_{f c t} / /[100]_{f c o}$, and

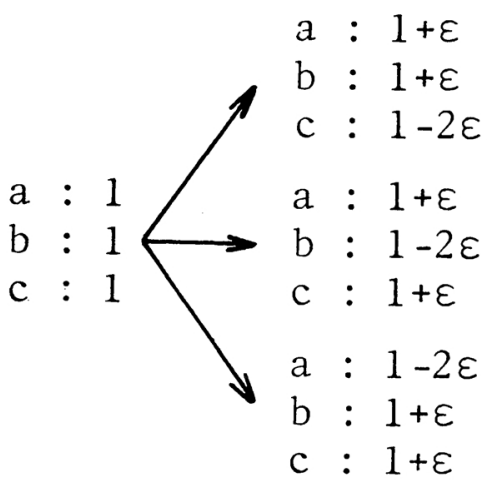

$\mathrm{fcc} \rightleftharpoons \mathrm{fct}(\mathrm{c} / \mathrm{a}<1)$

Fig. 9 Lattice correspondence in the $f c c \rightleftarrows f c t(c / a<1)$ transformation. 
two variants, which have the crystallographic relations of $[100]_{f c t} / /[001]_{f c o}$ and $[010]_{f c t} / /$ $[001]_{f c o}$, appear as the $f c o$ martensite as shown in Fig. 10. On the other hand, when $\delta$ becomes equal to $\varepsilon$ with falling temperature, the $f c o$ lattice is converted into the $f c t(c / a>1)$ one. Therefore, it is concluded that the $f c o \rightleftarrows f c t$ $(c / a>1)$ transformation is not martensitic and takes place by a continuous change in both $\varepsilon$ and $\delta$. In this case, we have a change in axial length given by $(c, b, a)=(1+2 \varepsilon, 1-\delta, 1-$ $2 \varepsilon+\delta) \rightarrow(1+2 \varepsilon, 1-\varepsilon, 1-\varepsilon)=(c, a, a)$. The orientation relation between two lattices is $[001]_{f c o} / /[001]_{f c t}$ and the axial ratio is given by $(1+2 \varepsilon) /(1-\varepsilon)=1+3 \varepsilon>1$. Figure 10 shows the lattice correspondence during the $f c t \rightarrow f c o \rightarrow f c t$ transformation. The crystallographic reversibility is preserved during the $f c t(c / a<1) \rightleftarrows f c o$ transformation, but is not always preserved through the $f c t \rightleftarrows f c o \rightleftarrows f c t$ transformation ${ }^{(13)}$. In view of the $f c t(c / a<1) \rightleftarrows f c t(c / a>1)$ lattice transformation observed in the In-(13-15 at \%) Sn alloys, a change in axial length is given by $(1+\varepsilon, 1+\varepsilon, 1-2 \varepsilon) \rightleftarrows(1+2 \varepsilon, 1-\varepsilon, 1-\varepsilon), \quad$ with reference to the $f c t(c / a<1) \rightleftarrows f c o \rightleftarrows f c t(c / a>1)$ transformation. In this case, a lattice deformation corresponding to the Bain lattice distortion is given by both a constraction by $2 \varepsilon$ along the $b$-axis and an expansion by $\varepsilon$ along the $a$ and the $c$-axis in the $f c t(c / a<1)$ lattice, and thus we have a relation $[100]_{f c t: c / a<1} / /[001]_{f c t: c / a>1}$. According to the resultant lattice correspondence shown in Fig. 11, two variants can exist

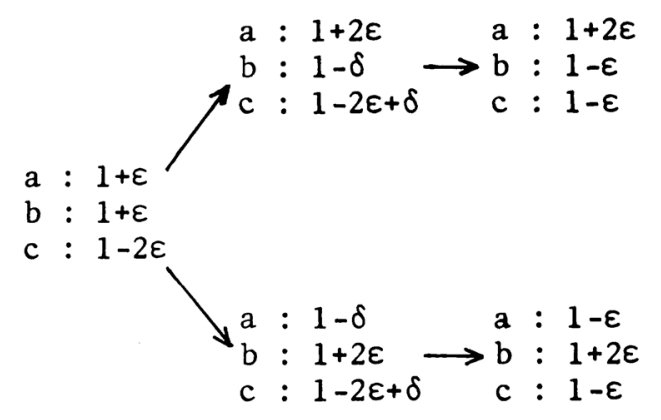

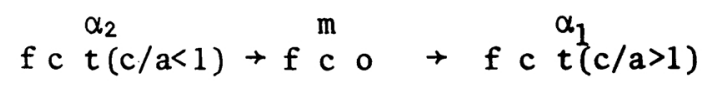

Fig. 10 Lattice correspondence in the $f c t(c / a<1) \rightarrow$ $f c o \rightarrow f c t(c / a>1)$ transformation. in the $f c t(c / a>1)$ lattice. After the $f c t(c / a>1) \rightarrow$ $f c t(c / a<1)$ reverse transformation, there are three variants of the $f c t(c / a<1)$ lattice, and as a result new bands corresponding to each variant are expected to appear. In fact, new bands are observed on a crystal surface, as shown in Photo. 7. The detail about the $f c t(c / a>1) \rightarrow$ $f c t(c / a<1)$ transformation is described in other papers $^{(12)(15)}$, although the reason why the old bands remain in the high-temperature $f c t(c)$ $a<1)$ phase could not be explained clearly. Finally the lattice correspondence discussed on the basis of the Bain lattice distortion was found to be consistent with the experimental result in each phase transformation. The propriety of this transformation mechanism, i.e. Bain-like deformation, has been discussed in terms of the soft phonon mode $e^{(16)}$ and also on the basis of the Landau theory of phase transformation $^{(11)(17)}$.

\section{Surface morphology of the low-temperature phase (martensite)}

Optical and X-ray topographic observations, together with the analysis of Laue photographs, have showed that a banded structure appears on the alloy surfaces after phase transformation and is due to $\{110\}$ transformation twinning. In this section, taking into consideration of the crystallographic relation between the banded structure and the lattice deformation during the phase transformation, relevant twin planes are determined on the basis of the Sapriel theory ${ }^{(18)}$ used for ferroelastic materials.

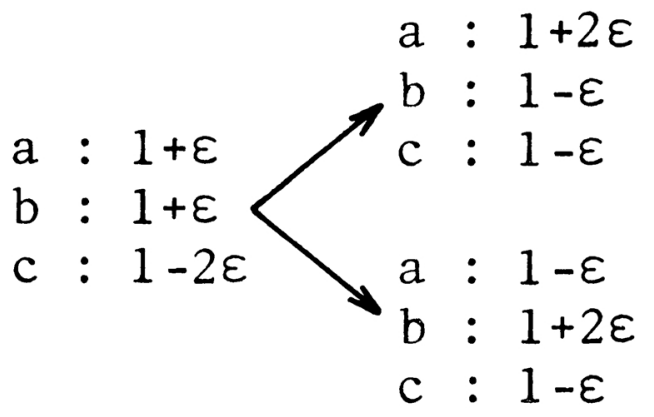

$\operatorname{fct}(c / a<1) \stackrel{f}{f} \operatorname{ct}(c / a>1)$

Fig. 11 Lattice correspondence in the $f c t(c / a<1) \rightleftarrows$ $f c t(c / a>1)$ transformation. 
Firstly we describe the theory briefly. Sapriel introduced a condition for determination of twin planes under two assumptions as follows: (1) A crystal is regarded as an elastic continuum, and (2) displacements caused by spontaneous strains in neighboring domains (i.e. variants) are equal to each other on a twin plane. The condition is given by

$$
S_{i j} X_{i} X_{j}=S_{i j}^{\prime} X_{i} X_{j}(i, j=1,2,3)
$$

where $S_{i j}$ and $S_{i j}^{\prime}$ are components of strain tensor $\{S\}$ denoting the spontaneous strains of neighboring domains and $X_{i}\left(X_{j}\right)$ is a component of a vector which denotes an arbitrary point within a crystal of paraelastic phase, i.e. a parent phase. When the strain tensors associated with phase transformation are known, twin planes concerning each phase transformation can be calculated by the eq. (1).

It has been previously shown that the phase transformations in indium-rich solid solutions are strain-induced ones and the low-temperature phases are ferroelastic ${ }^{(17)}$. The strain tensors associated with the $f c c \rightleftarrows f c t(c / a>1)$ and $f c c \rightleftarrows f c t(c / a<1)$ phase transformations are similar, and, with reference to the Bain lattice distortion previously described, they are given by

$$
\begin{aligned}
& S_{1}=\left(\begin{array}{ccc}
-2 \varepsilon & 0 & 0 \\
0 & \varepsilon & 0 \\
0 & 0 & \varepsilon
\end{array}\right), \quad S_{2}=\left(\begin{array}{ccc}
\varepsilon & 0 & 0 \\
0 & -2 \varepsilon & 0 \\
0 & 0 & \varepsilon
\end{array}\right) \\
& \text { and } S_{3}=\left(\begin{array}{ccc}
\varepsilon & 0 & 0 \\
0 & \varepsilon & 0 \\
0 & 0 & -2 \varepsilon
\end{array}\right),
\end{aligned}
$$

where there are three strain tensors corresponding to three variants in the $f c t$ phase and a sign of $\varepsilon$ is positive for the $f c t(c / a<1)$ phase and negative for the $f c t(c / a>1)$ one. Now, when $S_{1}$ and $S_{2}$ are chosen, the eq. (1) is reduced to

$$
-3 X_{1}^{2}+3 X_{2}^{2}=-3\left(X_{1}+X_{2}\right)\left(X_{1}-X_{2}\right)=0,
$$

so that two solutions, $X_{1}=X_{2}$ and $X_{1}=-X_{2}$, are obtained. These solutions show twin planes to be parallel to $(110)_{f c c}$ or $(1 \overline{10})_{f c c}$ planes. Finally two combinations from three spontaneous strains make us to have six possible twin planes parallel to $\{110\}$ planes in the $f c t$ phase.

Here, the $f c t(c / a<1) \rightleftarrows f c o$ transformation is discussed. According to the crystallographic correspondence between two crystal structures, there are two strain tensors which are represented by

$$
S_{1}=\left(\begin{array}{ccc}
-\varepsilon-\delta & 0 & 0 \\
0 & \varepsilon & 0 \\
0 & 0 & \delta
\end{array}\right) \text { and } S_{2}=\left(\begin{array}{ccc}
\varepsilon & 0 & 0 \\
0 & -\varepsilon-\delta & 0 \\
0 & 0 & \varepsilon
\end{array}\right) \text {. }
$$

In this case, only one combination of $S_{1}$ and $S_{2}$ is possible and the solutions are $X_{1}=X_{2}$ and $X_{1}=-X_{2}$. Thus the twin planes are parallel to the (110) planes or to the (110) planes. The spontaneous strains associated with the $f c t$ $(c / a<1) \rightleftarrows f c t(c / a>1)$ transformation are given by

$$
S_{1}=\left(\begin{array}{ccc}
-2 \varepsilon & 0 & 0 \\
0 & \varepsilon & 0 \\
0 & 0 & \varepsilon
\end{array}\right) \quad \text { and } \quad S_{2}=\left(\begin{array}{ccc}
\varepsilon & 0 & 0 \\
0 & -2 \varepsilon & 0 \\
0 & 0 & \varepsilon
\end{array}\right)
$$

In this case the twin planes are parallel to the $(110)_{f c t: c / a<1}$ planes or to the $(1 \overline{1} 0)_{f c t: c / a<1}$ planes in the $f c t(c / a>1)$ phase, being similar to the case of the $f c t(c / a<1) \rightleftarrows f c o$ phase transformation. These results mentioned above are essentially consistent with the observed relief morphology. This shows that the Sapriel theory is available for the analysis of the phase transformations in indium-rich solid solutions. Finally, it is concluded that the phase transformations in indium-rich alloys take place by the Bain-like lattice distortion and that martensites show a banded structure due to the $\{110\}$ transformation twinning on account of relaxation of transformation strains.

\section{REFERENCES}

(1) M. Hansen: Constitution of binary alloys, McGraw-Hill, New York, (1958).

(2) E. A. Shunk: Constitution of binary alloys, McGraw-Hill, New York, (1969).

(3) C. Tysack and G. V. Raynor: Trans. Faraday Soc., 50 (1954), 675.

(4) K. Yonemitsu: J. Phys. Soc. Japan, 21 (1966), 1231.

(5) L. Guttman: Trans. AIME, 188 (1950), 1472.

(6) J. S. Bowles, C. S. Barrett and L. Guttman: Trans. AIME, 188 (1950), 1478.

(7) A. Nagasawa: J. Phys. Soc. Japan, 30 (1971), 1200.

(8) A. Nagasawa: Phys. Status Solidi, (a) 8 (1971), 531.

(9) O. Nittono and Y. Koyama: J. Japan Inst.Metals, 42 (1978), 56 (in Japanese). 
(10) Y. Koyama and O. Nittono: J. Japan Inst. Metals, 43 (1979), 262 (in Japanese).

(11) Y. Koyama and O. Nittono: J. Japan Inst. Metals, 43 (1979), 696 (in Japanese).

(12) O. Nittono, H. Iwasaki and Y. Koyama: J. Japan Inst. Metals, 44 (1980) 899 (in Japanese).

(13) Y. Koyama, T. Ukena and O. Nittono: J. Japan Inst. Metals, 44 (1980), 1431 (in Japanese).

(14) O. Nittono and Y. Koyama: Sci. Rep. RITU,
A29, suppl. 1 (1981), 53.

(15) H. Iwasaki, O. Nittono and Y. Koyama: J. Japan Inst. Metals, 45 (1981), 667 (in Japanese).

(16) Y. Koyama and O. Nittono: J. Japan Inst. Metals, 45 (1981), 869 (in Japanese).

(17) O. Nittono and Y. Koyama: Jpn. J. Appl. Phys., 21 (1982) 680.

(18) J. Sapriel: Phys. Rev. B12 (1975) 5128.

(19) K. Aizu: J. Phys. Soc. Japan, 27 (1969) 387. 